\title{
Genetic Susceptibility of Alzheimer's Disease in East Asia
}

Can. J. Neurol. Sci. 2011; 38: 394-395

\begin{abstract}
Alzheimer's disease (AD) is the most common adult-onset dementia. Molecular genetic research has provided a wealth of information regarding the etiology of this disease. Identification and functional characterization of autosomal dominant mutations in the amyloid precursor protein gene and the presenilin genes 1 and 2 have contributed substantially to our understanding of the biological mechanisms leading towards neurodegeneration in AD. Nonetheless, a large portion of the genetic etiology remains unresolved, especially that of the more common or "sporadic" forms of AD. While substantial efforts have been invested in the identification of genetic risk factors underlying "sporadic" AD, the strongest established risk factor remains the $\varepsilon 4$ allele of the apolipoprotein $\mathrm{E}$ gene ${ }^{1}$.
\end{abstract}

Early genetic studies often investigated one or few single nucleotide polymorphisms at a time. Their success was considerably limited due to a variety of factors, including the absence of linkage disequilibrium between the marker tested and the underlying functional variant. More recently developed technologies have since allowed genome-wide studies to utilize the underlying linkage disequilibrium pattern, in haplotypebased approaches, in order to capture a wider amount of genetic information. Notwithstanding, the positive results obtained in some of these recent genome-wide studies often prove difficult to replicate because the original findings are 1) false-positives; 2) attributable to heterogeneity at the allelic, mutational, or populational level; 3) influenced by differences in study design or statistical analysis ${ }^{1}$. A regularly updated overview of genomic studies in $\mathrm{AD}$ can be found on the Alzgene website (www.alzgene.org) ${ }^{2}$. The following genes are on the top ten list: APOE_e2/3/4, CLU, PICALM, EXOC3L2 , BIN1 , CR1 , SORL1, GWA_14q32.13, TNK1, IL8.

In this issue of the Journal, Hao et al present a meta-analysis of mitochondrial aldehyde dehydrogenase (ALDH2) gene polymorphism in an AD cohort from East $\mathrm{Asia}^{3}$. As well, ALDH2 is one of the most important enzymes in human alcohol metabolism. The ALDH2*2 or "oriental variant" functions as a dominant negative, greatly reducing enzymatic activity in heterozygotes and abolishing activity in homozygotes. The ALDH $2 * 2$ was probably carried by Han Chinese as they spread throughout East Asia. The highest frequency of this allele can be seen in Southeast China, but also occurs in most areas of China, Japan, Korea, Mongolia, and Vietnam with progressively lower frequencies compared to Southeast China. This allele is associated with serious disorders such as alcoholic liver disease, colorectal cancer, and esophageal cancer, and is best known for protection against alcoholism ${ }^{4}$. Some basic research suggests that the level of ALDH2 activity may be related to agedependant neurodegeneration accompanying memory loss ${ }^{5}$.

Hao et al do not present new data per se, but rather try to address a controversy as to whether ALDH $2 * 2$ is associated with $\mathrm{AD}$ risk in East Asians by performing a meta-analysis. ${ }^{3}$ They included in their analysis four papers on $\mathrm{ALDH} 2 * 2$ that described retrospective or nested case-control studies, which included sufficient information from genotype counts to allow estimating the odds ratio at a $95 \%$ confidence interval. The main advantage of meta-analysis is increased statistical power and cost-effectiveness. However, there are flaws in combining genotypes or results of different studies. In the present case, population stratification is an issue, since in one study there is deviation from the Hardy-Weinberg equilibrium test. One can have a reasonably good idea of the effect of population stratification by simply looking at the frequency of ALDH2*2 carriers in controls, which can vary from $29 \%$ (South Korea) to $46 \%$ (China). In addition, the base populations are different since one is from Japan, one is from South Korea and two are from China. Also, one study is population-based and three are hospital-based, which can give an ascertainment bias. Another issue that has to be addressed is the publication bias that is bound to affect any meta-analysis, since it is well recognized that positive associations are easier to publish than negative associations. Hence, this may have affected the overall selection process, driving other unpublished negative studies out. One may also be concerned with subgroup analysis, which may give rise to false-positive results. This issue is to be kept in mind when considering the conclusion of the present study as to the effect reported specifically in the males. Finally, association analysis can detect small genetic influences, but even if a difference is real, $\mathrm{p}$ values do not indicate biologic importance. Thus, a meta-analysis of whole groups of similar studies does not necessarily increase confidence in the reality of findings in the individual studies.

Alzheimer's disease is now a major health burden on the aging population worldwide, be it in Canada or in East Asian countries. Immigration has also brought large populations of East Asians in North America, and it is important to study the specific environmental or genetic risk factors that may contribute to AD across ethnic groups. The data presented in the Journal by Hao et al is an effort at pooling several sets of available studies to address one specific genetic risk factor of AD in East Asians. ${ }^{3}$ The issue is not settled yet, however. Since there are elements to suggest a common genetic pool in East Asia, several prospective cohorts of harmonized sets of data would certainly improve the quality of futures results. This type of cohort would be available for rapid replication of the findings presented in this study, and would also support the more recently designed genome-wide approaches. 


\section{REFERENCES}

1. Brouwers N, Sleegers K, Van Broeckhoven C. Molecular genetics of Alzheimer's disease: an update. Ann Med. 2008;40(8):562-83.

2. Bertram L, McQueen MB, Mullin K, Blacker D, Tanzi RE. Systematic meta-analyses of Alzheimer disease genetic association studies: the AlzGene database. Nat Genet. 2007;39 (1): 17-23.

3. Hao P-P, Chen Y-G, Wang J-L, Wang XL, Zhang Y. Meta-analysis of ALDH2 gene polymorphism and Alzheimer's disease in East Asians. Can J Neuro Sci. 2011;38(3):500-6.
4. Li H, Borinskaya S, Yoshimura $\mathrm{K}$, et al. Refined geographic distribution of the oriental ALDH2*504Lys (nee 487Lys) variant. Ann Hum Genet. 2009;73(Pt 3):335-45.

5. Ohsawa I, Nishimaki K, Murakami Y, Suzuki Y, Ishikawa M, Ohta $\mathrm{S}$. Age-dependent neurodegeneration accompanying memory loss in transgenic mice defective in mitochondrial aldehyde dehydrogenase 2 activity. J Neurosci. 2008; 28(24):6239-49. 